\title{
La salud en un México en transición*
}

Juan Ramón de la Fuente.(1)

$\mathrm{E}$ s para mí un honor y una distinción que mucho aprecio el haber sido invitado a dictar la Conferencia Magistral Miguel E Bustamante, en el marco de este $X$ Congreso de Investigación en Salud Pública. Y lo es por varias razones: por la figura que se evoca, por la calidad profesional de quienes en años anteriores han sustentado esta Conferencia, y también por lo que el Instituto Nacional de Salud Pública significa en el ámbito de la salud, la educación y la investigación en nuestro país.

Don Miguel Bustamante es, sin duda, una figura señera en nuestra historia de la salud. Oaxaqueño de origen, llevaba la salud pública en la sangre. Su abuelo fue un sanitarista distinguido, que trabajó incansablemente por mejorar las condiciones de vida de los pobladores del Itsmo de Tehuantepec. Pero la sangre es sólo un componente en la vida de una persona. El doctor Bustamante la complementó en la Universidad de Johns Hopkins, de donde egresó como el primer mexicano con un doctorado en salud pública. Posteriormente impulsó con gran vigor, a través de la Escuela de Salud Pública, la formación de recursos humanos cada vez mejor calificados en este campo.

Su trabajo es fundamental para entender los avances que, en su época, se alcanzaron ante problemas complejos como lo eran la fiebre amarilla, la viruela, el mal del pinto, la lepra, la oncocercosis, el paludismo y la tos ferina, por mencionar algunos. Fue Presidente de la Academia Nacional de Medicina y su trabajo fue reconocido internacionalmente por la Organización Mundial de la Salud, la Oficina Sanitaria Panamericana y el Fondo de las Naciones Unidas para la Infancia.

Académico riguroso, Bustamante dejó un legado importante de publicaciones en artículos diversos y li- bros que son esenciales para entender la historia de la salubridad y de la salud pública en México. También la revista Salud Pública de México puede considerarse como un fruto de la trascendente obra del doctor Bustamante.

Justamente por ello conviene iniciar el tema haciendo una firme reivindicación de la salud pública en nuestro país, de quienes la forjaron y de quienes la han seguido cultivando, depurando y enriqueciendo a lo largo del tiempo, porque gracias a esa visión y a esa perspectiva, México ha logrado avanzar sustancialmente en sus niveles de salud. Sin duda subsisten algunos problemas, y se van presentando otros que son novedosos, pero lo que es indiscutible es que hoy contamos con una mejor infraestructura sanitaria, con recursos humanos más capacitados y mejor preparados para enfrentar, tanto los nuevos retos como los viejos desafíos, desde una posición más sólida en lo conceptual y en lo operativo.

Conviene también recordar que este año la Secretaría de Salud cumplirá sus primeros 60 años desde su creación lo cual hace, asimismo oportuno, reflexionar sobre lo que ha ocurrido en nuestro país a lo largo de estas décadas y, de manera objetiva, bien documentada, tratar de ubicar en su justa dimensión los avances y los rezagos; los problemas y las respuestas que ante éstos ha dado el Sistema Nacional de Salud, con sus imperfecciones, pero también con sus indiscutibles fortalezas.

Entender el proceso salud-enfermedad no es sencillo, y un factor determinante que en México ha jugado un papel esencial ha sido la dinámica poblacional. En 1960, por ejemplo, la tasa de mortalidad era 11.5, 40 años más tarde era 4.5; la esperanza de vida al nacer * Conferencia Magistral "D r. Miguel E. Bustamante", dictada por el doctor Juan Ramón de la Fuente durante el X Congreso de Investigación en Salud
Pública, celebrado del 5 al 7 de marzo de 2003, en el Instituto N acional de Salud Pública, Cuernavaca, Morelos, México.

(1) Rector de la Universidad Nacional Autónoma de México. 
era de 57 años y ahora lo es de poco más de 75; había 78000 médicos y hoy hay más de 130 000, y las unidades médicas que eran 870 son ahora más de 19000.

Sin pretender ser exhaustivo en el análisis, lo que resulta innegable es que entre 1930 y el año 2000 la población total aumentó seis veces al pasar de 17 a cerca de 100 millones de habitantes; la población urbana aumentó 13 veces, al grado que hoy tres de cada cuatro mexicanos vivimos en zonas urbanas. En 1960 sólo $20 \%$ de la población total habitaba en localidades mayores de 100000 habitantes y hoy lo hace la mitad de la población.

El aumento desorbitado de la población ocasionó que el Estado enfrentara una creciente limitación de recursos, y el mayor ritmo de crecimiento en las grandes ciudades y la presión social por cubrir sus necesidades propiciaron que en las zonas rurales más pobres se acentuaran los rezagos. Una gráfica sencilla en la que se combinen las tasas de crecimiento anual del producto interno bruto, las tasas de crecimiento de la población y la evolución que ha tenido el ingreso per cápita, puede ilustrar con toda claridad este fenómeno. Pero también conviene señalar que, gracias al esfuerzo del Sistema Nacional de Salud, el crecimiento poblacional ha entrado ya en una dinámica diferente. La pirámide demográfica nacional del año 2000 muestra, por primera vez en la historia, que su base, es decir la que se refiere al grupo poblacional entre 0 y 4 años, es más estrecha que la que corresponde al grupo de edad entre los 5 y los 9 años. Estamos entrando pues a un proceso en el que la población potencialmente productiva habrá de superar a la población dependiente. Este fenómeno representa una gran oportunidad para nuestro desarrollo económico y social.

El tema que nos ocupa permite, asimismo, recorrer a grandes pasos algunos eventos que son importantes para entender mejor el momento que vivimos en materia de salud y valorar, con rigor pero sin sesgos ideológicos o coyunturales, nuestro sistema de salud.

Los cambios en la salud de las poblaciones toman tiempo, y a veces es más prudente darle continuidad a aquellas acciones que han funcionado bien, que modificarlas precipitadamente. La introducción a principios del siglo XIX de la vacuna contra la viruela por Balmis, por ejemplo, logra su mejor expresión casi 150 años después, cuando se registró en San Luis Potosí el último caso de viruela en México. Y por cierto, no hay que olvidar que la erradicación de la viruela aquí se logró 26 años antes de su erradicación total en el mundo.

Otro avance importante fue la erradicación de la poliomielitis en 1995. El último caso se presentó en Jalisco en 1990. Esto se logró gracias a un enorme esfuerzo de muchos años, de muchas generaciones, y acredita nuestra magnífica tradición de combate a las enfermedades prevenibles por vacunación: desde 1991 no hay casos de difteria; el sarampión está prácticamente controlado -aunque hubo un brote en el año 2000-y la tos ferina y el tétanos son casi inexistentes. Esta larga historia, que es un buen ejemplo de lo que puede ser una política de Estado, se ha reforzado con el tiempo, y desde 1998 la inclusión de nuevos biológicos nos permite tener uno de los esquemas de vacunación gratuitos más completos del mundo.

Ciertamente los retos en salud hoy han tomado un nuevo y para muchos insospechado cariz. El bioterrorismo nos ha forzado a revivir la vacuna contra la viruela, que parecía ya no tener mucho sentido. El hecho es que hoy la viruela se cierne nuevamente como una grave amenaza que podría implicar una de las reemergencias más dramáticas de una enfermedad en la historia universal de la salud. La pregunta que surge entonces es: cuándo debemos realmente dejar de vacunar a la población contra algún padecimiento aparentemente erradicado; y desde luego, también preguntarnos a conciencia si estamos preparados para hacerle frente a la emergencia sanitaria que el bioterrorismo puede plantearnos de manera más o menos súbita. No sólo la viruela, el ántrax o el botulismo, sino también por medio de agentes químicos. Yo tengo mis dudas al respecto, sustentadas en lo que los dos mexicanos calificados como inspectores por las Naciones Unidas me han comentado recientemente.

Pero en todo caso me refiero al asunto del bioterrorismo para ilustrar cómo en materia de salud no parece haber batallas que puedan ganarse definitivamente; en el mejor de los casos pueden ganarse tentativamente. Todo el capítulo de las enfermedades reemergentes nos deja esa lección, una lección de humildad. Y sirve también para mostrar cómo los retos al sistema de salud son a veces imprevisibles y no obstante, lo que se requiere, lo que la sociedad espera, es una rápida capacidad de respuesta.

Otra pregunta relevante es: ¿cómo sabemos si realmente hemos avanzado en nuestras condiciones de salud? Si esta pregunta la llevamos a una comunidad rural, marginada, en Oaxaca o en Guerrero, sería difícil dar una respuesta alentadora. Los promedios nacionales, lo sabemos bien, encubren realidades dolorosas, y por ello es fundamental que contemos con información veraz: global, nacional, regional y local; que se profundice en el Federalismo que tanto trabajo ha costado impulsar, a pesar de que el Pacto Federal que nos constituyó como nación independiente se suscribió hace más de 180 años.

En nuestro país, con enormes esfuerzos, se ha avanzado también de manera estimable en el rubro de 
la información, y se ha consolidado un sistema cada vez más sólido, auditable, cuyos márgenes de error han venido disminuyendo y que nos han permitido empezar a armar ese gran rompecabezas, con indicadores objetivos, construidos en las áreas de servicio y también a través de los estudios de campo.

Una herramienta esencial han sido las encuestas, y en ello el Instituto de Salud Pública ha jugado un papel fundamental. Las encuestas nacionales de salud, la de nutrición, las de adicciones (realizadas por el Instituto Nacional de Psiquiatría), las de cobertura de vacunación, todas ellas nos han permitido reforzar ese sistema de información y hay al respecto publicaciones serias. Sepúlveda, Gutiérrez Trujillo y Kumate publicaron hace algunos años La salud en cifras, y recientemente junto con Roberto Tapia y Miguel Angel Lezana publicamos La información en salud, un libro que da cuenta del esfuerzo realizado al año 2000, incluyendo datos del Sistema Nacional de Vigilancia Epidemiológica, que es otro instrumento que se ha venido depurando e incorporando nuevos elementos tecnológicos que lo hacen cada vez más eficiente.

Desde 1995 se cuenta con un Comité Nacional de Vigilancia Epidemiológica, que permitió la creación del Sistema Unico de Información para la Vigilancia Epidemiológica, homologando en todas las instituciones del Sector Salud el contenido, la periodicidad, las formas de notificar y registrar la información, etcétera. Fue gracias a ello que se pudo obtener el certificado de erradicación de la poliomielitis. Dos años después se incorporó al Sistema la Red Hospitalaria de Vigilancia Epidemiológica, que incluye ya a más de 130 unidades médicas y registra información relativa a infecciones nosocomiales, tuberculosis, defectos de cierre del tubo neural y diabetes, entre otras.

Un cambio aparentemente menor pero que en realidad no lo fue, consistió en incorporar las cifras de mortalidad, que antes se manejaban exclusivamente como estadísticas vitales, al Sistema Epidemiológico y Estadístico de las Defunciones, lo que permitió empezar a conocer la situación de la mortalidad en el país con tan sólo dos meses de diferencia. Cuando cruzamos esta información con el INEGI en 1998, la correlación fue superior a $90 \%$. Otro cambio trascendente consistió en incorporar el laboratorio al Sistema de Vigilancia Epidemiológica, con lo que se fortaleció sustancialmente la vigilancia epidemiológica de problemas como el SIDA y la tuberculosis.

En suma, podemos decir sin temor a equivocarnos, que el sistema de información en salud del que hoy disponemos en México, siempre susceptible de mejorarse, es un sistema confiable.
Otra faceta interesante que ha tenido el Sistema Nacional de Salud en los últimos años, se ha puesto en relieve al confrontar problemas de salud emergentes o reemergentes. Empezaré por el cólera. A principios de 1991, las autoridades peruanas informaron la ocurrencia de un brote de diarrea aguda en pobladores de localidades costeras. Dicho evento fue el inicio de la séptima pandemia de cólera en el continente americano.

La respuesta de nuestro Sistema de Salud fue oportuna y eficiente. En junio de ese mismo año, después de más de un siglo de silencio, se confirmó el diagnóstico de cólera en un hombre de 57 años de edad en el Estado de México. Su arribo a esa localidad fue un tanto inesperado: no ocurrió en la frontera sur ni en los puertos náuticos o aéreos como se esperaba. Un grupo de expertos de la Secretaría de Salud logró establecer que unos días antes habían estado cerca del lugar algunos sudamericanos, uno de los cuales al parecer tenía diarrea. Se localizó también cerca de ahí una pista aérea clandestina, que aparentemente estos sudamericanos habían utilizado con fines inciertos pero imaginables.

A pesar de las dimensiones que alcanzó el problema, nada tienen que ver con los estragos que el cólera ocasionó en nuestro país un siglo antes. La séptima pandemia de cólera en México tuvo dos periodos: el primero de 1991 a 1997, caracterizado por el aumento de los casos en los años nones y la reducción de los mismos en los años pares. La letalidad aumentó en 1993 a $1.8 \%$, y gracias a la respuesta del sistema de salud al año siguiente volvió a bajar de $1.5 \%$ que es el límite internacionalmente aceptado. En 1995 tuvimos 16430 casos y la información, lejos de minimizarse, se difundió ampliamente desde la Secretaría de Salud, lo que propició una mucho mayor participación social. En octubre de 1997 se registró la última defunción por cólera. El segundo periodo, de 1998 a la actualidad, se ha caracterizado por la continua reducción en el número de casos, al grado que en 2001 hubo un solo caso, con lo cual México salió de la lista de países infectados por el cólera. Las consecuencias de la pandemia han sido dolorosas y muy lamentables sobre todo por las pérdidas humanas que causó, pero proporcionalmente fueron mucho menores a los daños ocasionados en casi todos los otros países afectados.

El error ahora sería bajar la guardia; festinar que ya no tenemos cólera, porque no podemos garantizar que la pandemia haya concluido y, sobre todo, porque la octava pandemia, debida a Vibrio cholerae 0139 ya ronda en Asia y recientemente se volvió a identificar la presencia del vibrión en las costas de Chile.

Un aspecto singular de nuestro sistema de salud, ha sido su capacidad de respuesta en los casos de de- 
sastres naturales. Dos huracanes de gran magnitud se presentaron consecutivamente en 1995: Opalo en septiembre y Roxana en octubre. En las zonas afectadas hubo un repunte importante en el número de los casos de cólera. Se afectaron los sistemas de abasto de agua, de drenaje, de recolección de desechos, etcétera, lo cual ocasionó una fuerte contaminación en agua y alimentos. Pero las brigadas de salud impidieron que el desastre hidrometeorológico se convirtiera en un desastre sanitario. A partir de esa dramática experiencia se empezó a desarrollar un modelo de atención a las poblaciones afectadas por estos desastres y poco después fue adoptado, a sugerencia de la Organización Panamericana de la Salud, en varios países centroamericanos como consecuencia del huracán Mitch. Las brigadas de salud jugaron un papel extraordinario en Centroamérica y ayudaron, como pocos, a mitigar los efectos devastadores de ese huracán. Hubo reconocimientos y agradecimientos explícitos de los gobiernos de Costa Rica, Cuba, El Salvador, Guatemala, Honduras, Nicaragua y Venezuela.

El Sistema Nacional de Salud ha mostrado además su capacidad de coordinación y de liderazgo. Durante el huracán Paulina, por ejemplo, las primeras acciones del Plan DN-3 las encabezó la Secretaría de Salud. El modelo funcionó también en 1998 durante las inundaciones en la costa y la Sierra Madre de Chiapas; en 1999 durante los terremotos de Puebla y Oaxaca; en la depresión tropical que afectó a Tabasco, Campeche y Veracruz, y en los más recientes casos en Yucatán, Nayarit y Colima. El área afectada por esos fenómenos es mayor a la superficie de varios países, y la población atendida en su conjunto sobrepasa los 10 millones de personas.

Las adicciones constituyen otro problema emergente que se ha enfrentado razonablemente, considerando su complejidad. Los estudios poblacionales que se iniciaron hace más de 10 años han permitido dimensionar la magnitud del problema, y han sido fundamentales para poder contar con información veraz sobre el tema. Sin estas encuestas, seguramente seguiríamos en el discurso hueco, retórico, acomodándolo a las circunstancias. Aquí también, el Sistema de Vigilancia Epidemiológica nos ha permitido recabar información sobre la conducta y las características de la población afectada, su contexto, los patrones de consumo y su ubicación geográfica. Nuestro prestigio ante el National Institute of Drug Abuse es incuestionable. Recientemente fue nombrada para dirigirlo una mexicana formada en México y colaboradora en muchos de los esfuerzos pioneros que se hicieron en la materia. Paradojas del destino: una médica mexicana dirige la principal institución contra las drogas de los Estados
Unidos de América. La capacidad de la Secretaría de Salud para negociar y acotar la publicidad de industrias económicamente muy poderosas como son las del tabaco y el alcohol, ha fortalecido también la autoridad del sistema de salud.

El liderazgo de nuestro país en el campo de las adicciones y la veracidad con la que se ha manejado la información hizo posible que, a propuesta de México, junto con Portugal y Suecia, se realizara en 1998 una Asamblea General Extraordinaria de las Naciones Unidas para tratar de unir esfuerzos y contrarrestar el grave problema global que representa el consumo de drogas. Los avances no son lo satisfactorio que quisiéramos pero la lucha debe seguir.

A finales de la década de los setenta apareció una nueva y devastadora enfermedad: el VIH/SIDA. El último informe sobre esta epidemia mundial muestra que el año pasado el SIDA cobró más de tres millones de vidas y que otros cinco millones se infectaron, lo que eleva a 42 millones el número de personas que actualmente viven con el virus del VIH en el mundo. En México se estima que son del orden de 150000 los que actualmente viven con el virus. La mayoría no lo sabe.

El reto es tanto o más serio que el que significó en su momento erradicar la viruela. Por eso es también oportuno evocar hoy la figura y la obra de Bustamante. Requerimos una política más vigorosa para detener esta enfermedad, aunque ciertamente se han adoptado medidas importantes. En 1986 se creó CONASIDA; 11 años después, en 1997 se creó FONSIDA, lo que permitió en su momento que todas las mujeres embarazadas con VIH y todos los menores de 15 años de edad afectados tuvieran acceso a medicamentos y esquemas de atención razonables. Se promovió la participación de la sociedad y se incorporaron las organizaciones sociales a las tareas institucionales, articulando consensos y desarrollando proyectos conjuntos encaminados a la prevención y control de la enfermedad.

Desde que apareció el SIDA en México se inició el combate frontal a las fuerzas oscurantistas que preferían "no hablar del asunto", combate que prosiguió durante varios años hasta que se les venció, aunque no se les convenció. Hubo grandes dificultades, críticas, demandas y escándalos pero se afrontaron uno a uno y hoy el tema ya no es tabú, aunque subsiste la discriminación para muchos enfermos y se percibe un nuevo embate moralista que en nada ayuda a la solución del problema.

En 1999 México alcanzó la Presidencia de la Junta Directiva de ONUSIDA, desde donde se establecieron las bases para el desarrollo de la vigilancia de segunda generación, que México ya había iniciado dos años 
antes, para reducir el subregistro y el retraso en la notificación.

Todos los anteriores son ejemplos que ilustran, en este México de transición, algunos aspectos de nuestro Sistema Nacional de Salud, que muestran su evolución y su fortaleza, sin pretender ocultar sus rezagos ni sus debilidades. Los logros alcanzados han sido posibles gracias a que hoy tenemos más y mejores recursos humanos, educados con métodos y herramientas modernas para hacerle frente a estos problemas. Cursos de maestría y doctorado de alto nivel que hace pocos años no había; este mismo Congreso que apenas inaugura su décima edición, y otros esfuerzos como la Residencia en Epidemiología Aplicada que se inició con el apoyo de los Centros para la Prevención y el Control de Enfermedades de Atlanta, y que cuenta desde 1995 con reconocimiento de la Facultad de Medicina de la Universidad Nacional Autónoma de México, así como la consolidación del Consejo Nacional de Salud Pública, reconocido por la Academia Nacional de Medicina. Todos estos y otros más son expresión inobjetable del esfuerzo que se ha hecho para profesionalizar a la salud pública en nuestro país.

Se decía al inicio de esta conferencia que la dinámica poblacional en México, gracias a los programas de planificación familiar y salud reproductiva, empieza a mostrar una disminución en el índice de dependencia demográfica; es decir, en la relación que hay entre la población dependiente y la población en edad productiva. Este índice, según las proyecciones, alcanzará su nivel más bajo en el año 2020 y a partir de ahí empezará nuevamente a subir, a expensas del crecimiento en la población de personas mayores de 65 años de edad. En los próximos años alcanzaremos pues el tan anhelado bono demográfico. Todo indica que, si no se afloja en los programas de salud reproductiva, la población infantil continuará con su tendencia descendente respecto a su participación en la población total, y la población en edades productivas se incrementará en casi $50 \%$ en términos absolutos. En muchas ciudades el bono demográfico ya empezó, puesto que la transición poblacional en nuestro país no es un proceso homogéneo y existen diferencias marcadas entre las entidades federativas en el inicio del proceso, su duración, el tiempo de desfase entre la declinación de la mortalidad y la fecundidad, etcétera.
El hecho es que este bono demográfico nos brinda una gran oportunidad para dar el salto que hemos estado buscando desde hace varios años y alcanzar mejores niveles de vida para la mayoría de los mexicanos, si somos capaces de generar los empleos que se requieren y de fortalecer los compromisos sociales del Estado, fundamentalmente en materia de educación y salud.

La disminución del índice de dependencia demográfica permite que haya una mejoría sustancial en la distribución del ingreso, pero habrá que afinar bien el foco en las nuevas prioridades: de la educación básica a la educación media superior y superior; de la prevención de enfermedades transmisibles a la prevención de las enfermedades crónicas, sin descuidar los avances alcanzados y construyendo sobre éstos las nuevas estrategias que se requieren.

La salud está en la agenda política de los gobiernos y México no es la excepción. El viejo debate entre Estado liberal y Estado social está resurgiendo con fuerza en muchos países en el contexto del mundo interdependiente en el que estamos inmersos. México necesita, desde mi perspectiva, un Estado fuerte que no eluda sus compromisos sociales. Son éstos los que le dan razón de ser y auténtica legitimidad. Un Estado débil en un país como México dejará inevitablemente a la deriva a millones de personas, que sin mejor salud y mayor educación difícilmente podrán acceder a niveles de vida dignos y productivos.

Concluyo reiterando que los ideales del doctor Bustamante siguen vivos, así como los de otros grandes maestros en los campos de la medicina y la salud pública, que fueron ejemplo de lo que México puede llegar a ser, a lo que debemos aspirar. Tenemos en nuestras propias raíces una fuente inagotable de inspiración. Aprovechemos la tradición y fortaleza de nuestras instituciones públicas; siguen siendo la mejor expresión social de lo que los mexicanos hemos sido capaces de construir a lo largo de nuestra propia historia.

\section{Agradecimientos}

Agradezco al doctor Roberto Tapia Conyer su colaboración en la elaboración de este texto. 